Bull. Korean Math. Soc. 50 (2013), No. 6, pp. 2053-2059

http://dx.doi.org/10.4134/BKMS.2013.50.6.2053

\title{
SHARPENED FORMS OF THE SCHWARZ LEMMA ON THE BOUNDARY
}

\author{
BÜLENT NAFi ÖRNEK
}

\begin{abstract}
In this paper, a boundary version of the Schwarz lemma is investigated. We obtain more general results at the boundary. Also, new inequalities of the Schwarz lemma at boundary is obtained and the sharpness of these inequalities is proved.
\end{abstract}

\section{Introduction}

The classical Schwarz lemma states that a holomorphic function $f$ mapping the unit disc $D$ into itself, with $f(0)=0$, satisfies the inequality $|f(z)| \leq|z|$ for any point $z \in D([3]$, p. 381).

Let $f$ be a holomorphic function on $D, f(0)=1$ and $|f(z)-\varepsilon|<\varepsilon$ for $|z|<1$, where $\varepsilon$ is real number and $\frac{1}{2}<\varepsilon \leq 1$.

Consider the functions

$$
\vartheta(z)=\frac{f(z)-\varepsilon}{\varepsilon}
$$

and

$$
\omega(z)=\frac{\vartheta(z)-\vartheta(0)}{1-\overline{\vartheta(0)} \vartheta(z)} .
$$

$\vartheta(z)$ and $\omega(z)$ are holomorphic functions in the $\operatorname{disc} D,|\omega(z)|<1$ for $|z|<1$ and $\omega(0)=0$. Therefore, from the Schwarz lemma, we obtain

$$
|f(z)| \leq \varepsilon \frac{1+|z|}{\varepsilon+(1-\varepsilon)|z|}
$$

and

$$
\left|f^{\prime}(0)\right| \leq \frac{2 \varepsilon-1}{\varepsilon} .
$$

Equality is achieved in (1.1) (for some nonzero $z \in D$ ) or in (1.2) if and only if $f(z)$ is the function of the form $f(z)=\varepsilon \frac{1+z e^{i \theta}}{\varepsilon+(1-\varepsilon) z e^{i \theta}}$, where $\theta$ is a real number.

Received January 2, 2013; Revised April 5, 2013.

2010 Mathematics Subject Classification. 30C80.

Key words and phrases. Schwarz lemma on the boundary, holomorphic function, angular limit and derivative, Julia-Wolff-Lemma. 
It is an elementary consequence of Schwarz lemma that if $f$ extends continuously to some boundary point $z_{0}$ with $\left|z_{0}\right|=1$, and if $\left|f\left(z_{0}\right)\right|=1$ and $f^{\prime}\left(z_{0}\right)$ exists, then $\left|f^{\prime}\left(z_{0}\right)\right| \geq 1$, which is known as the Schwarz lemma on the boundary. Applying inequality (5) in ([2], p. 330) to the function $\frac{f(z)}{z}$, we arrive at the following generalization of the Schwarz lemma [2]:

$$
|f(z)| \leq|z| \frac{|z|+\left|f^{\prime}(0)\right|}{1+|z|\left|f^{\prime}(0)\right|}, z \in D \text {. }
$$

If, in addition, the function $f$ has an angular limit $f\left(z_{0}\right)$ at $z_{0} \in \partial D,\left|f\left(z_{0}\right)\right|=$ 1 , then by Julia-Wolff-Lemma the angular derivative $f^{\prime}\left(z_{0}\right)$ exists and $1 \leq$ $\left|f^{\prime}\left(z_{0}\right)\right| \leq \infty[5]$. Then, passing to the angular limit in (1.3), we arrive at the boundary Schwarz lemma [4]

$$
\left|f^{\prime}\left(z_{0}\right)\right| \geqslant \frac{2}{1+\left|f^{\prime}(0)\right|}
$$

In (1.3) for real $z$ and in the left-hand-side inequality in (1.4) for $z_{0}=1$, equality occurs for the function $f(z)=z(z+a) /(1+a z), 0 \leq a \leq 1$.

It follows that

$$
\left|f^{\prime}\left(z_{0}\right)\right| \geqslant 1
$$

with equality only if $f$ is of the form $f(z)=z e^{i \theta}, \theta$ real.

Moreover, if $f(z)=c_{p} z^{p}+c_{p+1} z^{p+1}+\cdots$, then

$$
\left|f^{\prime}\left(z_{0}\right)\right| \geqslant p+\frac{1-\left|c_{p}\right|}{1+\left|c_{p}\right|}
$$

It follows that

$$
\left|f^{\prime}\left(z_{0}\right)\right| \geqslant p
$$

with equality only if $f$ is of the form $f(z)=z^{p} e^{i \theta}, \theta$ real.

Previously, R. Osserman, examined sharp Schwarz inequality at the boundary (see [4]). Afterwards, the Schwarz inequality that has been obtained by V. Dubinin is strengthened (see [1]).

We will obtain more general results at the boundary. In the following theorems, new inequalities of Schwarz inequality at the boundary are obtained and the sharpness of these inequalities is proved.

Theorem 1.1. Let $f$ be a holomorphic function in the disc $D, f(0)=1$ and let $|f(z)-\varepsilon|<\varepsilon$ for $|z|<1$, where $\varepsilon$ is real number and $\frac{1}{2}<\varepsilon \leq 1$. Further assume that, for some $z_{0} \in \partial D, f$ has an angular limit $f\left(z_{0}\right)$ at $z_{0}, f\left(z_{0}\right)=2 \varepsilon$. Then

$$
\left|f^{\prime}\left(z_{0}\right)\right| \geq \varepsilon(2 \varepsilon-1) .
$$

The equality in (1.8) holds if and only if

$$
f(z)=\varepsilon \frac{1+z e^{i \theta}}{\varepsilon+(1-\varepsilon) z e^{i \theta}},
$$


where $\theta$ is a real number.

Proof. Let

$$
\omega(z)=\frac{\vartheta(z)-\vartheta(0)}{1-\overline{\vartheta(0)} \vartheta(z)} .
$$

The function $\omega(z)$ is holomorphic in the unit disc $D,|\omega(z)|<1$ for $|z|<1$, $\omega(0)=0$ and $\left|\omega\left(z_{0}\right)\right|=1$ for $z_{0} \in \partial D$.

From (1.5), we obtain

$$
1 \leq\left|\omega^{\prime}\left(z_{0}\right)\right|=\frac{1-|\vartheta(0)|^{2}}{\left|1-\overline{\vartheta(0)} \vartheta\left(z_{0}\right)\right|^{2}}\left|\vartheta^{\prime}\left(z_{0}\right)\right|=\frac{1-\left(\frac{1-\varepsilon}{\varepsilon}\right)^{2}}{\left(1-\frac{1-\varepsilon}{\varepsilon}\right)^{2}} \frac{\left|f^{\prime}\left(z_{0}\right)\right|}{\varepsilon}=\frac{\left|f^{\prime}\left(z_{0}\right)\right|}{(2 \varepsilon-1) \varepsilon}
$$

Therefore, we have

$$
\left|f^{\prime}\left(z_{0}\right)\right| \geq \varepsilon(2 \varepsilon-1) .
$$

If $\left|f^{\prime}\left(z_{0}\right)\right|=\varepsilon(2 \varepsilon-1)$ from (1.10) and $\left|\omega^{\prime}\left(z_{0}\right)\right|=1$, we obtain

$$
f(z)=\varepsilon \frac{1+z e^{i \theta}}{\varepsilon+(1-\varepsilon) z e^{i \theta}} .
$$

Theorem 1.2. Under the same assumptions as in Theorem 1.1, we have

$$
\left|f^{\prime}\left(z_{0}\right)\right| \geq \frac{2 \varepsilon(2 \varepsilon-1)^{2}}{(2 \varepsilon-1)+\varepsilon\left|f^{\prime}(0)\right|} .
$$

The inequality (1.11) is sharp with equality for the function

$$
f(z)=\varepsilon \frac{1+2 a z+z^{2}}{\varepsilon+a z+(1-\varepsilon) z^{2}},
$$

where $a=\frac{\varepsilon\left|f^{\prime}(0)\right|}{(2 \varepsilon-1)}$ is arbitrary number from $[0,1]($ see $(1.2))$.

Proof. Let $\omega(z)$ be the same as in the proof of Theorem 1.1. Using inequality (1.4) for the function $\omega(z)$, we obtain

$$
\begin{aligned}
\frac{2}{1+\left|\omega^{\prime}(0)\right|} & \leq\left|\omega^{\prime}\left(z_{0}\right)\right|=\frac{\left|f^{\prime}\left(z_{0}\right)\right|}{(2 \varepsilon-1) \varepsilon} \\
\frac{2}{1+\frac{\varepsilon\left|f^{\prime}(0)\right|}{(2 \varepsilon-1)}} & \leq \frac{\left|f^{\prime}\left(z_{0}\right)\right|}{(2 \varepsilon-1) \varepsilon}
\end{aligned}
$$

and

$$
\left|f^{\prime}\left(z_{0}\right)\right| \geq \frac{2 \varepsilon(2 \varepsilon-1)^{2}}{(2 \varepsilon-1)+\varepsilon\left|f^{\prime}(0)\right|} .
$$

Now, we shall show that the inequality (1.11) is sharp. Choose arbitrary $a \in$ $[0,1]$. Let

$$
f(z)=\varepsilon \frac{1+2 a z+z^{2}}{\varepsilon+a z+(1-\varepsilon) z^{2}} .
$$


Then

$$
f^{\prime}(z)=\varepsilon \frac{(2 a+2 z)\left(\varepsilon+a z+(1-\varepsilon) z^{2}\right)-(a+2(1-\varepsilon) z)\left(1+2 a z+z^{2}\right)}{\left(\varepsilon+a z+(1-\varepsilon) z^{2}\right)^{2}}
$$

and

$$
f^{\prime}(1)=2 \varepsilon \frac{2 \varepsilon-1}{1+a}
$$

Since $\mathrm{a}=\frac{\varepsilon\left|f^{\prime}(0)\right|}{(2 \varepsilon-1)},(1.11)$ is satisfied with equality.

If $f(z)=1+c_{p} z^{p}+c_{p+1} z^{p+1}+\cdots, p \geq 1$, is a holomorphic function in $D$ and $|f(z)-\varepsilon|<\varepsilon$ for $|z|<1$, where $\varepsilon$ is real number and $\frac{1}{2}<\varepsilon \leq 1$, then

$$
|f(z)| \leq \varepsilon \frac{1+|z|^{p}}{\varepsilon+(1-\varepsilon)|z|^{p}}
$$

and

$$
\left|c_{p}\right| \leq \frac{2 \varepsilon-1}{\varepsilon}
$$

Theorem 1.3. Let $f(z)=1+c_{p} z^{p}+c_{p+1} z^{p+1}+\cdots, p \geq 1$, be a holomorphic function in the disc $D$ and let $|f(z)-\varepsilon|<\varepsilon$ for $|z|<1$, where $\varepsilon$ is real number and $\frac{1}{2}<\varepsilon \leq 1$. Further assume that, for some $z_{0} \in \partial D, f$ has an angular limit $f\left(z_{0}\right)$ at $z_{0}, f\left(z_{0}\right)=2 \varepsilon$. Then

$$
\left|f^{\prime}\left(z_{0}\right)\right| \geq p \varepsilon(2 \varepsilon-1)
$$

with equality (1.13) if and only if $f(z)$ is the function of the form

$$
f(z)=\varepsilon \frac{1+z^{p} e^{i \theta}}{\varepsilon+(1-\varepsilon) z^{p} e^{i \theta}},
$$

where $\theta$ is a real number.

Proof. Using inequality $\left|f^{\prime}\left(z_{0}\right)\right| \geq p$ for the function $\omega(z)$, we obtain

$$
\left|\omega^{\prime}\left(z_{0}\right)\right| \geq p .
$$

So,

$$
p \leq\left|\omega^{\prime}\left(z_{0}\right)\right|=\frac{1-|\vartheta(0)|^{2}}{\left|1-\overline{\vartheta(0)} \vartheta\left(z_{0}\right)\right|^{2}}\left|\vartheta^{\prime}\left(z_{0}\right)\right|=\frac{1-\left(\frac{1-\varepsilon}{\varepsilon}\right)^{2}}{\left(1-\frac{1-\varepsilon}{\varepsilon}\right)^{2}} \frac{\left|f^{\prime}\left(z_{0}\right)\right|}{\varepsilon}=\frac{\left|f^{\prime}\left(z_{0}\right)\right|}{(2 \varepsilon-1) \varepsilon} .
$$

If $\left|f^{\prime}\left(z_{0}\right)\right|=p \varepsilon(2 \varepsilon-1)$ from (1.14) and $\left|\omega^{\prime}\left(z_{0}\right)\right|=p$, we obtain

$$
f(z)=\varepsilon \frac{1+z^{p} e^{i \theta}}{\varepsilon+(1-\varepsilon) z^{p} e^{i \theta}} .
$$


Theorem 1.4. Under the same assumptions as in Theorem 1.3, we have

$$
\left|f^{\prime}\left(z_{0}\right)\right| \geq \varepsilon(2 \varepsilon-1)\left(p+\frac{2 \varepsilon-1-\varepsilon\left|c_{p}\right|}{2 \varepsilon-1+\varepsilon\left|c_{p}\right|}\right) .
$$

The inequality (1.15) is sharp with equality for the function

$$
f(z)=\varepsilon \frac{1+b z+b z^{p}+z^{p+1}}{\varepsilon+\varepsilon b z+(1-\varepsilon)\left(z^{p+1}+b z^{p}\right)},
$$

where $b=\frac{\varepsilon\left|c_{p}\right|}{(2 \varepsilon-1)}$ is an arbitrary number on $[0,1]($ see $(1.12))$.

Proof. Using inequality (1.6) for the function $\omega(z)$, we obtain

$$
p+\frac{1-\left|a_{p}\right|}{1+\left|a_{p}\right|} \leq\left|\omega^{\prime}\left(z_{0}\right)\right|=\frac{\left|f^{\prime}\left(z_{0}\right)\right|}{(2 \varepsilon-1) \varepsilon},
$$

where $\left|a_{p}\right|=\frac{\left|\omega^{(p)}(0)\right|}{p !}=\frac{\varepsilon\left|c_{p}\right|}{(2 \varepsilon-1)}$. Therefore, we take

$$
p+\frac{1-\frac{\varepsilon\left|c_{p}\right|}{(2 \varepsilon-1)}}{1+\frac{\varepsilon\left|c_{p}\right|}{(2 \varepsilon-1)}} \leq \frac{\left|f^{\prime}\left(z_{0}\right)\right|}{(2 \varepsilon-1) \varepsilon}
$$

and

$$
\left|f^{\prime}\left(z_{0}\right)\right| \geq \varepsilon(2 \varepsilon-1)\left(p+\frac{2 \varepsilon-1-\varepsilon\left|c_{p}\right|}{2 \varepsilon-1+\varepsilon\left|c_{p}\right|}\right) .
$$

The equality in (1.15) is obtained for the function

$$
f(z)=\varepsilon \frac{1+b z+b z^{p}+z^{p+1}}{\varepsilon+\varepsilon b z+(1-\varepsilon)\left(z^{p+1}+b z^{p}\right)},
$$

as show simple calculations.

Theorem 1.5. Let $f(z)=1+c_{p} z^{p}+c_{p+1} z^{p+1}+\cdots, p \geq 1$, be a holomorphic function in the disc $D$ and let $|f(z)-\varepsilon|<\varepsilon$ for $|z|<1$, where $\varepsilon$ is real number and $\frac{1}{2}<\varepsilon \leq 1$. Further assume that, for some $z_{0} \in \partial D, f$ has an angular limit $f\left(z_{0}\right)$ at $z_{0}, f\left(z_{0}\right)=2 \varepsilon$. Let $b_{1}, b_{2}, \ldots, b_{n}$ be zeros of the function $f(z)-1$ in $D$ that are different from zero. Then we have the inequality

$$
\left|f^{\prime}\left(z_{0}\right)\right| \geq \varepsilon(2 \varepsilon-1)\left(p+\sum_{k=1}^{n} \frac{1-\left|b_{k}\right|^{2}}{\left|z_{0}-b_{k}\right|^{2}}+\frac{(2 \varepsilon-1) \prod_{k=1}^{n}\left|b_{k}\right|-\varepsilon\left|c_{p}\right|}{(2 \varepsilon-1) \prod_{k=1}^{n}\left|b_{k}\right|+\varepsilon\left|c_{p}\right|}\right) .
$$

In addition, the equality in (1.16) occurs for the function

$$
f(z)=\varepsilon \frac{1+z^{p} \prod_{k=1}^{n} \frac{z-b_{k}}{1-\overline{b_{k}} z}}{\varepsilon+(1-\varepsilon) z^{p} \prod_{k=1}^{n} \frac{z-b_{k}}{1-\overline{b_{k}} z}},
$$

where $b_{1}, b_{2}, \ldots, b_{n}$ are positive real numbers. 
Proof. Consider the functions

$$
\omega(z)=\frac{\vartheta(z)-\vartheta(0)}{1-\overline{\vartheta(0)} \vartheta(z)}, B(z)=\prod_{k=1}^{n} \frac{z-b_{k}}{1-\overline{b_{k}} z} .
$$

$\omega(z)$ and $B(z)$ are holomorphic functions in $D$, and $|\omega(z)|<1,|B(z)|<1$ for $|z|<1$. By the maximum principle for each $z \in D$, we have

$$
|\omega(z)|<|B(z)|
$$

The composite function

$$
\Phi(z)=\frac{\omega(z)}{B(z)}=\frac{\vartheta(z)-\vartheta(0)}{1-\overline{\vartheta(0)} \vartheta(z)} \frac{1}{\prod_{k=1}^{n} \frac{z-b_{k}}{1-\overline{b_{k}} z}}
$$

is a holomorphic function in $D$, and $|\Phi(z)|<1$ for $|z|<1, \Phi(0)=0$ and $\left|\Phi\left(z_{0}\right)\right|=1$ for $z_{0} \in \partial D$.

Moreover, it can be seen that

$$
\frac{z_{0} \omega^{\prime}\left(z_{0}\right)}{\omega\left(z_{0}\right)}=\left|\omega^{\prime}\left(z_{0}\right)\right| \geq\left|B^{\prime}\left(z_{0}\right)\right|=\frac{z_{0} B^{\prime}\left(z_{0}\right)}{B\left(z_{0}\right)} .
$$

Besides, with the simple calculations, we take

$$
\left|B^{\prime}\left(z_{0}\right)\right|=\frac{z_{0} B^{\prime}\left(z_{0}\right)}{B\left(z_{0}\right)}=\sum_{k=1}^{n} \frac{1-\left|b_{k}\right|^{2}}{\left|z_{0}-b_{k}\right|^{2}} .
$$

Using inequality (1.6) for the function $\Phi(z)$, we obtain

$$
p+\frac{1-\left|d_{p}\right|}{1+\left|d_{p}\right|} \leq\left|\Phi^{\prime}\left(z_{0}\right)\right|=\left|\frac{z_{0} \omega^{\prime}\left(z_{0}\right)}{\omega\left(z_{0}\right)}-\frac{z_{0} B^{\prime}\left(z_{0}\right)}{B\left(z_{0}\right)}\right|=\left\{\left|\omega^{\prime}\left(z_{0}\right)\right|-\left|B^{\prime}\left(z_{0}\right)\right|\right\},
$$

where $\left|d_{p}\right|=\frac{\left|\Phi^{(p)}(0)\right|}{p !}$.

Since $\left|d_{p}\right|=\frac{\left|\Phi^{(p)}(0)\right|}{p !}=\frac{\varepsilon\left|c_{p}\right|}{(2 \varepsilon-1) \prod_{k=1}^{n}\left|b_{k}\right|}$, we may write

$$
\begin{aligned}
1-\frac{\varepsilon\left|c_{p}\right|}{(2 \varepsilon-1) \prod_{k=1}^{n}\left|b_{k}\right|} & \leq \frac{1-\left(\frac{1-\varepsilon}{\varepsilon}\right)^{2}}{\left(1-\frac{1-\varepsilon}{\varepsilon}\right)^{2}} \frac{\left|f^{\prime}\left(z_{0}\right)\right|}{\varepsilon}-\sum_{k=1}^{n} \frac{1-\left|b_{k}\right|^{2}}{\left|z_{0}-b_{k}\right|^{2}} \\
& =\frac{\left|f^{\prime}\left(z_{0}\right)\right|}{(2 \varepsilon-1) \prod_{k=1}^{n}\left|b_{k}\right|}-\sum_{k=1}^{n} \frac{1-\left|b_{k}\right|^{2}}{\left|z_{0}-b_{k}\right|^{2}} .
\end{aligned}
$$

Therefore, we take inequality (1.16). 
The equality in (1.16) is obtained for the function

$$
f(z)=\varepsilon \frac{1+z^{p} \prod_{k=1}^{n} \frac{z-b_{k}}{1-\overline{b_{k}} z}}{\varepsilon+(1-\varepsilon) z^{p} \prod_{k=1}^{n} \frac{z-b_{k}}{1-\overline{b_{k}} z}},
$$

as show simple calculations.

Acknowledgement. The author would like to thank the referee for his/her constructive comments and suggestions on the earlier version of this paper.

\section{References}

[1] V. N. Dubinin, On the Schwarz inequality on the boundary for functions regular in the disk, J. Math. Sci. (N. Y.) 122 (2004), no. 6, 3623-3629.

[2] G. M. Golusin, Geometric Theory of Functions of Complex Variable, 2nd edn., Moscow 1966.

[3] A. I. Markushevich, Theory of Functions of a Complex Variable. Volume I, 1965.

[4] R. Osserman, A sharp Schwarz inequality on the boundary, Proc. Math. Soc. 128 (2000), no. $12,3513-3517$

[5] Ch. Pommerenke, Boundary Behaviour of Conformal Maps, Springer-Verlag, Berlin, 1992.

Department of Mathematics

Gebze Institute of Technology

Gebze-Kocaeli 41400, Turkey

E-mail address: nornek@gyte.edu.tr 